\title{
PLANAR SECTIONS THROUGH THREE-DIMENSIONAL LINE-SEGMENT PROCESSES
}

\author{
Sascha DJamal MatThes ${ }^{\bowtie, 1}$ AND Dietrich StOYAN $^{2}$ \\ ${ }^{1}$ Institut für Keramik, Glas- und Baustofftechnik, Technische Universität Bergakademie Freiberg, D-09596 \\ Freiberg, Germany; ${ }^{2}$ Institut für Stochastik, Technische Universität Bergakademie Freiberg, D-09596 Freiberg, \\ Germany \\ e-mail: Sascha-Djamal.Matthes@ikgb.tu-freiberg.de \\ (Received September 25, 2013; revised January 14, 2014; accepted January 14, 2014)
}

\begin{abstract}
This paper studies three-dimensional segment processes in the framework of stochastic geometry. The main objective is to find relations between the characteristics of segment processes such as orientation- and lengthdistribution, and characteristics of their sections with planes. Formulae are derived for the distribution of segment lengths on both sides of the section plane and corresponding orientations, where it is permitted that there are correlations between the angles and lengths of the line-segments.
\end{abstract}

Keywords: fibre process, fibre-reinforced materials, line-segment process, stereology, stochastic geometry.

\section{INTRODUCTION}

Segment processes are stochastic models for random systems of line-segments randomly scattered in space. They belong to the more general class of fibre processes, the mathematical theory of which was developed by Joseph Mecke and coworkers (Mecke and Nagel, 1980; Mecke and Stoyan, 1980b; Chiu et al., 2013).

These processes find important applications in the context of fibre-reinforced materials, where fibres, which can be often modelled as line-segments of negligible thickness, are embedded in a matrix of more or less homogeneous material.

In the now classical papers mentioned above planar sections played an important role. Such sections produce systems of fibre-plane intersection points, which can be statistically analysed with the aim to get information on the spatial fibre system. This setting belongs to the field of stereology, and a classical formula there is

$$
L_{V}=2 N_{A},
$$

where $L_{V}$ is the mean total fibre length per unit volume and $N_{A}$ the number of intersection points per unit area. (The formula holds true under the assumptions of statistical homogeneity or stationarity and isotropy, see also Chiu et al., 2013.)

Planar sections through segment processes appear in the context of fibre-reinforced materials, when axial tension is studied. Following Li et al. (1991) the intersections of fibres with a plane orthogonal to the tension axis are investigated. Additionally to the characteristics studied when stereology is of interest, also the residual lengths of the line-segments on both sides of the section plane are of importance in the mechanical calculations. (They have never been considered in the stereological context, since these lengths cannot be measured in the section plane.)

The present paper first explains a natural segment process model, following the pattern of Mecke and Stoyan (1980a). Then it derives formulae for the section process characteristics. Some of them have counterparts in the classical theory, while those related to the residual lengths are new, generalising results in Li et al. (1991), who considered the case of segments of constant length. Furthermore, formulae for the maximum and minimum residual segment length are derived since these characteristics play a role in calculations of the contribution of fibres to the mechanical strength in a composite material.

\section{MODEL DESCRIPTION}

This paper considers three-dimensional linesegment processes. A realisation of such a process is a set of randomly distributed line-segments in space. To characterise such a line-segment we use its top point (in the sense of the $z$-axis) $(x, y, z) \in \mathbb{R}^{3}$, the length $l>0$ and the angles $\lambda$ and $\beta$ denoting azimuth and polar angle of the line-segment. Since we are not interested in the sense of direction of the line-segment, with $\lambda \in[0,2 \pi]$ and $\beta \in\left[0, \frac{\pi}{2}\right]$ a line-segment is well defined. 


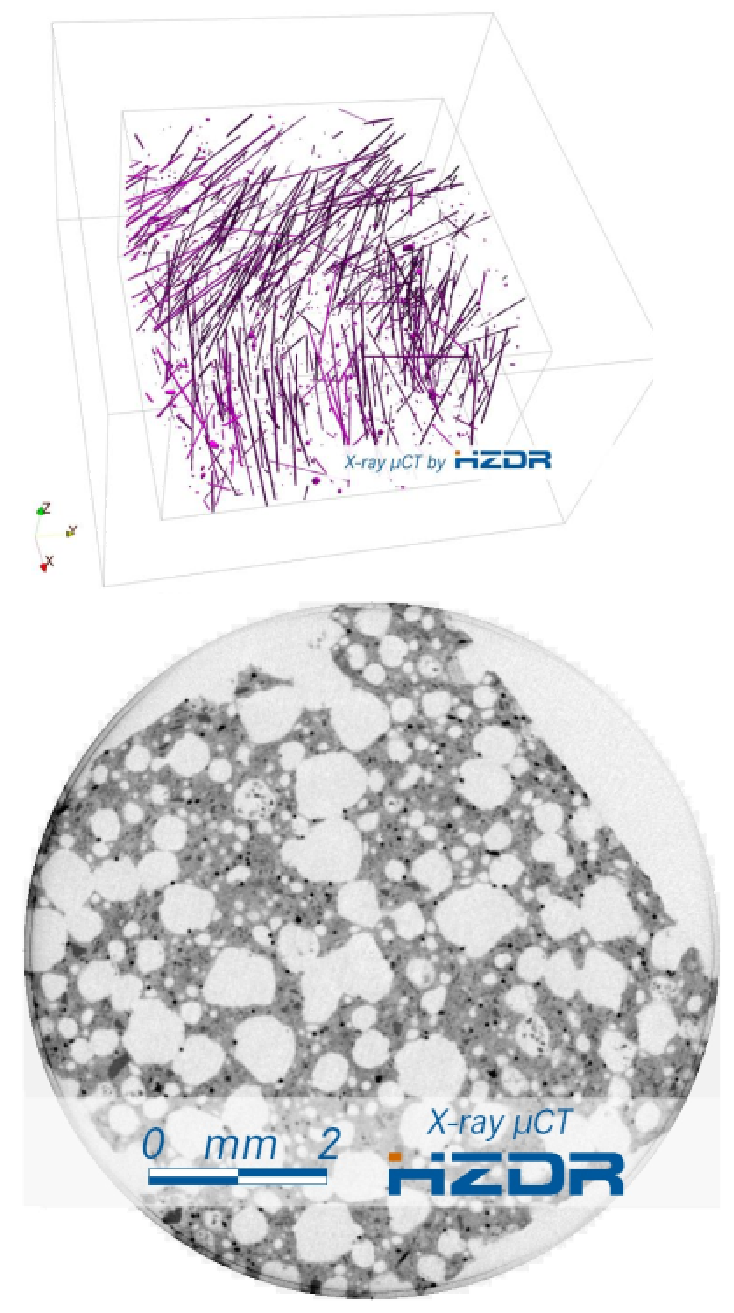

Figure 1. Computer-tomography-based measurements on fibre-reinforced autoclaved aerated concrete. The upper figure shows the three-dimensional distribution of fibres. The lower figure presents a planar section of reinforced material. The section points of the fibres (black points) form a random point pattern.

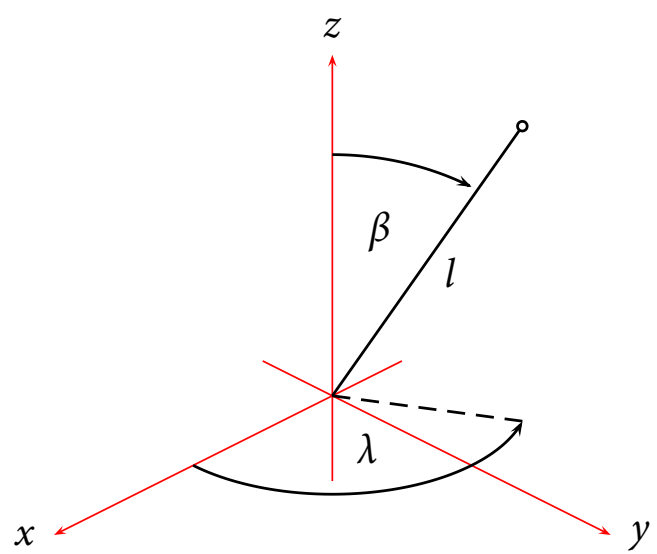

Figure 2. Geometrical representation of a line-segment which is shifted so that its endpoint meets the origin of the coordinate system.

A line-segment process is here represented as a marked point process $\Psi_{V}$ (for more on marked point processes see Chiu et al., 2013). Realisations of $\Psi_{V}$ can be written as sequences of marked points:

$$
\psi_{V}=\left\{\left[\left(x_{i}, y_{i}, z_{i}\right), l_{i}^{V}, \lambda_{i}^{V}, \beta_{i}^{V}\right]\right\},
$$

with $\left(x_{i}, y_{i}, z_{i}\right) \in \mathbb{R}^{3}, l_{i}^{V}>0, \lambda_{i}^{V} \in[0,2 \pi]$ and $\beta_{i}^{V} \in$ $\left[0, \frac{\pi}{2}\right]$. Moreover we introduce the stochastic variables of the polar angle, $B^{V}$, the azimuthal angle, $\Lambda^{V}$, and the fibre length, $L^{V}$, of a typical line-segment of $\Psi_{V}$.

Here and in the following we assume $\Psi_{V}$ to be stationary, i.e., the distribution of $\Psi_{V}$ is translation invariant. It is not assumed that the marked point process $\Psi_{V}$ has some specific distribution, e.g., a marked Poisson process. The results presented in this paper hold for every distribution of a stationary $\Psi_{V}$, where $B^{V}$ and $\Lambda^{V}$ are stochastically dependent (see page 57) or independent (see page 60) of $L^{V}$.

\section{ESSENTIAL PROPERTIES}

The distribution of the marked point process $\Psi_{V}$ is described by the following characteristics:

Table 1. Characteristics of the spatial marked point process $\Psi_{V}$

\begin{tabular}{ll}
\hline$N_{V}$ & $\begin{array}{l}\text { mean number of top points of } \\
\text { line-segments per unit volume }\end{array}$ \\
$F_{V, L, B, \Lambda}(l, \beta, \lambda)$ & $\begin{array}{l}\text { joint distribution function of } \\
\text { fibre length } L^{V} \text { and angles } \\
\\
B^{V} \text { and } \Lambda^{V} \text { of a typical line } \\
\text { segment }\end{array}$ \\
$F_{V, B}(\beta), F_{V, \Lambda}(\lambda)$, & $\begin{array}{l}\text { marginal distribution functions } \\
\text { of the stochastic variables } B^{V},\end{array}$ \\
$F_{V, L}(l)$ & $\Lambda^{V}$ and $L^{V}$. \\
\hline
\end{tabular}

The stochastic variables of the polar angle, $B^{V}$, and the azimuthal angle, $\Lambda^{V}$ may depend on the stochastic variable of the spatial fibre length, $L^{V}$.

In order to study the mechanical behavior of fibre-reinforced materials under axial tensions the intersection of a plane with a line-segment system is of peculiar interest. The mechanical effect of a fibre in a homogeneous material depends on the intersection angle and the length of the fibre under and over the plane respectively. Thus these quantities have to be studied. This approach appears in the classical papers by Li et al. (1991), Brandt (1985) and in subsequent work. However, in these papers the segment lengths are assumed to be constant.

Due to our homogeneity assumption we choose the intersecting plane to be the $(x, y)$-plane $S=\{(x, y, z) \in$ $\left.\mathbb{R}^{3}: z=0\right\}$. Intersection of the segments of $\Psi_{V}$ with 
$S$ yields the marked point process $\Psi_{A}$ of intersection points with the realizations

$$
\psi_{A}=\left\{\left[\left(\xi_{i}, \eta_{i}\right), r_{1, i}^{A}, r_{2, i}^{A}, \lambda_{i}^{A}, \beta_{i}^{A}\right]\right\}
$$

where $\left(\xi_{i}, \eta_{i}\right)$ are the intersection points, $r_{1, i}^{A}$ and $r_{2, i}^{A}$ the lengths of the upper and lower part of the segments respectively and $\lambda_{i}^{A}$ and $\beta_{i}^{A}$ the corresponding section angles. Due to stationarity of $\Psi_{V}$ also the marked point process $\Psi_{A}$ is stationary.

We introduce the stochastic variables of the polar section angle, $B^{A}$, the azimuthal section angle $\Lambda^{A}$ and the upper and lower part of the segment which belongs to the typical intersection point, $R_{1}^{A}$ and $R_{2}^{A}$ respectively. The basic constants and distribution functions of the marked point process $\Psi_{A}$ are shown in the following table:

Table 2. Characteristics of the planar marked point process $\Psi_{A}$

\begin{tabular}{ll}
\hline$N_{A}$ & $\begin{array}{l}\text { mean number of section } \\
\text { points of } \Psi_{A} \text { per unit area }\end{array}$ \\
$F_{A, R_{1}, R_{2}, \Lambda, B}\left(r_{1}, r_{2}, \lambda, \beta\right)$ & $\begin{array}{l}\text { joint distribution function } \\
\text { of upper and lower }\end{array}$ \\
& segment lengths and \\
& intersection angles $B^{A}, \Lambda^{A}$ \\
& at a typical section point \\
& marginal distribution \\
& functions of the upper and \\
& lower segment lengths \\
$F_{A, R_{1}, R_{2}}\left(r_{1}, r_{2}\right)$, & and the intersection \\
$F_{A, \Lambda}(\lambda), F_{A, B}(\beta)$ & angles $B^{A}$ and $\Lambda^{A}$. \\
\hline
\end{tabular}

\section{BASIC CHARACTERISTICS OF $\Psi_{A}$ AND RELATIONS TO $\Psi_{V}$}

\section{MAIN RESULTS}

The main objective at this point is to establish relations between the basic constants and distribution functions of $\Psi_{V}$ and those of $\Psi_{A}$. Due to the choice of the intersection plane $S$ and the definition of the azimuthal angle $\lambda$, the latter can be ignored.

We concentrate on relations between $N_{A}, N_{V}$ and the marginal distribution functions $F_{A, R_{1}, R_{2}, B}\left(r_{1}, r_{2}, \beta\right)$ and $F_{V, L, B}(l, \beta)$. The following general basic equation holds for $N_{A}, N_{V}>0, r_{1}, r_{2}>0$ and $\beta \in\left[0, \frac{\pi}{2}\right]$ :

$$
\begin{array}{r}
N_{A} F_{A, R_{1}, R_{2}, B}\left(r_{1}, r_{2}, \beta\right)= \\
=N_{V} \int_{0}^{\beta} \sin \beta^{\prime} \int_{0}^{\min \left\{r_{1}, r_{2}\right\}}\left(F_{V, L, B}\left(l+\max \left\{r_{1}, r_{2}\right\}, \beta^{\prime}\right)\right. \\
\left.\quad N_{V} \cos \beta \int_{0}^{\min \left\{r_{1}, r_{2}\right\}}\left(l, \beta^{\prime}\right)\right) \mathrm{d} l \mathrm{~d} \beta^{\prime} \\
\quad F_{V, L, B}\left(l+\max \left\{r_{1}, r_{2}\right\}, \beta\right) \\
\left.-F_{V, L, B}(l, \beta)\right) \mathrm{d} l . \quad \text { (4) }
\end{array}
$$

The relation of the corresponding probability density functions $f_{V, L, B}(l, \beta)$ and $f_{A, R_{1}, R_{2}, B}\left(r_{1}, r_{2}, \beta\right)$ is therefore

$$
N_{A} f_{A, R_{1}, R_{2}, B}\left(r_{1}, r_{2}, \beta\right)=N_{V} \cos \beta f_{V, L, B}\left(r_{1}+r_{2}, \beta\right) .
$$

Eq. 4 is the starting point for some important formulae. Let $\beta=\frac{\pi}{2}$ and $r_{1}, r_{2} \rightarrow \infty$. Then we obtain the intensity of $\Psi_{A}$ as

$$
N_{A}=N_{V} \mathbf{E}\left(L^{V} \cos B^{V}\right),
$$

where

$$
\mathbf{E}\left(L^{V} \cos B^{V}\right)=\int_{0}^{\frac{\pi}{2}} \int_{0}^{\infty} l \cos \beta f_{V, L, B}(l, \beta) \mathrm{d} l \mathrm{~d} \beta .
$$

This expression simplifies for the isotropic case and if $L^{V}$ and $B^{V}$ are stochastic independent, see Eq. 26.

With the latter relation we are able to determine $F_{A, R_{1}, R_{2}, B}$ if $N_{V}$ and $F_{V, L, B}$ are given. Furthermore, we obtain with Eq. 4, Eq. 6 and $\beta=\frac{\pi}{2}$ the joint distribution function

$$
F_{A, R_{1}, R_{2}}\left(r_{1}, r_{2}\right)=F_{A, R_{1}, R_{2}, B}\left(r_{1}, r_{2}, \frac{\pi}{2}\right)
$$

of the upper and lower segment lengths. These distributions exist if $\mathbf{E} \cos B^{V} \neq 0$, i.e., if the case of all fibres parallel to the section plane is excluded.

$$
\begin{aligned}
N_{A} F_{A, R_{1}, R_{2}}\left(r_{1}, r_{2}\right)= & \\
= & N_{V} \int_{0}^{\min \left\{r_{1}, r_{2}\right\}} \int_{0}^{\frac{\pi}{2}} \sin \beta^{\prime}\left(F_{V, L, B}\left(l+\max \left\{r_{1}, r_{2}\right\}, \beta^{\prime}\right)\right. \\
& \left.-F_{V, L, B}\left(l, \beta^{\prime}\right)\right) \mathrm{d} \beta^{\prime} \mathrm{d} l, \\
= & \frac{1}{\mathbf{E}\left(L^{V} \cos B^{V}\right)} \int_{0}^{\min \left\{r_{1}, r_{2}\right\}} \int_{0}^{\frac{\pi}{2}} \sin \beta^{\prime} . \\
& \left(F_{V, L, B}\left(l+r_{2}\right)=\right.
\end{aligned}
$$


with $0 \leq r_{1}, r_{2}<\infty$. We can determine the marginal distribution functions for the upper and lower segment lengths

$$
F_{A, R_{1}}\left(r_{1}\right)=\lim _{r_{2} \rightarrow \infty} F_{A, R_{1}, R_{2}}\left(r_{1}, r_{2}\right)
$$

and

$$
F_{A, R_{2}}\left(r_{2}\right)=\lim _{r_{1} \rightarrow \infty} F_{A, R_{1}, R_{2}}\left(r_{1}, r_{2}\right) .
$$

The stochastic variables $R_{1}^{A}$ and $R_{2}^{A}$ are identically distributed with the distribution function

$$
\begin{aligned}
& F_{A, R}(r)=F_{A, R_{1}}(r)=F_{A, R_{2}}(r)= \\
& \frac{1}{\mathbf{E}\left(L^{V} \cos B^{V}\right)} \int_{0}^{r} \int_{0}^{\frac{\pi}{2}} \sin \beta^{\prime}\left(F_{V, B}\left(\beta^{\prime}\right)\right. \\
&\left.\quad-F_{V, L, B}\left(l, \beta^{\prime}\right)\right) \mathrm{d} \beta^{\prime} \mathrm{d} l,
\end{aligned}
$$

for $r>0$ and $F_{V, B}(\beta)=\lim _{l \rightarrow \infty} F_{V, L, B}(l, \beta)$.

With Eqs. 6 and 4 and $r_{1}, r_{2} \rightarrow \infty$ we analogously get the distribution function of the section angle $B^{A}$

$$
\begin{aligned}
& F_{A, B}(\beta)= \\
& \frac{1}{\mathbf{E}\left(L^{V} \cos B^{V}\right)} \int_{0}^{\infty}\left(\int_{0}^{\beta} \sin \beta^{\prime}\left(F_{V, B}\left(\beta^{\prime}\right)-F_{V, L, B}\left(l, \beta^{\prime}\right)\right) \mathrm{d} \beta^{\prime}\right. \\
& \left.\quad+\cos \beta\left(F_{V, B}(\beta)-F_{V, L, B}(l, \beta)\right)\right) \mathrm{d} l .
\end{aligned}
$$

With Eqs. 6-9 we have explicit relations for the basic characteristics of $\Psi_{A}$. We can add also the probability density functions using Eqs. 6-9:

$$
\begin{aligned}
& f_{A, R_{1}, R_{2}}\left(r_{1}, r_{2}\right)= \\
& \frac{1}{\mathbf{E}\left(L^{V} \cos B^{V}\right)} \int_{0}^{\frac{\pi}{2}} \cos \beta f_{V, L, B}\left(r_{1}+r_{2}, \beta\right) \mathrm{d} \beta,
\end{aligned}
$$

and

$$
\begin{aligned}
& f_{A, B}(\beta)= \\
& \frac{1}{\mathbf{E}\left(L^{V} \cos B^{V}\right)} \int_{0}^{\infty} \int_{0}^{\infty} \cos \beta f_{V, L, B}\left(r_{1}+r_{2}, \beta\right) \mathrm{d} r_{1} \mathrm{~d} r_{2} .
\end{aligned}
$$

The length of the line-segments were assumed to be stochastically dependent on the angle $B^{V}$ throughout the above investigations. Eq. 4 shows that, consequently, the line-segment lengths $R_{1}^{A}$ and $R_{2}^{A}$ are stochastically dependent of the intersection angle $B^{A}$.

\section{SKETCH OF THE PROOF OF EQ. 4}

The proof follows the pattern of Mecke and Stoyan (1980a), which is also used in Mecke and Stoyan (1980c).

Let $S_{D}\left(t_{1}, t_{2}, b, c\right)$ be the expected number of segments $p_{i}=\left[\left(x_{i}, y_{i}, z_{i}\right), l_{i}^{V}, \lambda_{i}^{V}, \beta_{i}^{V}\right]$ of the marked point process $\Psi_{V}$ fulfilling the following conditions:

1. the line-segment $p_{i}$ intersects a given compact subset $D$ of the plane $S$,

2. the line-segment has intersection angles $\beta_{i}^{A} \in[0, b]$ with $b \in\left[0, \frac{\pi}{2}\right]$ and $\lambda_{i}^{A} \in[0, c]$ with $c \in[0,2 \pi]$,

3. the lengths of the segment above and below $S$ fulfil $r_{1, i}^{A} \leq t_{1}$ and $r_{2, i}^{A} \leq t_{2}$ with $t_{1}, t_{2}>0$.

The quantity $S_{D}\left(t_{1}, t_{2}, b, c\right)$ can be calculated in terms of $\Psi_{A}$ and $\Psi_{V}$, and equating the corresponding terms yields Eq. 4.

The Campbell theorem (see, e.g., Chiu et al., 2013) applied to $\Psi_{A}$ yields simply

$$
S_{D}\left(t_{1}, t_{2}, b, c\right)=N_{A} F_{A, R_{1}, R_{2}, B, \Lambda}\left(t_{1}, t_{2}, b, c\right)
$$

for $t_{1}, t_{2}>0, b \in\left[0, \frac{\pi}{2}\right]$ and $c \in[0,2 \pi]$.

In terms of $\Psi_{V}, S_{D}\left(t_{1}, t_{2}, b, c\right)$ can be expressed as

$$
S_{D}\left(t_{1}, t_{2}, b, c\right)=\mathbf{E}\left(\sum_{p_{i} \in \psi_{V}} f\left(p_{i}, t_{1}, t_{2}, b, c\right)\right)
$$

for $t_{1}, t_{2}>0, b \in\left[0, \frac{\pi}{2}\right]$ and $c \in[0,2 \pi], p_{i}=$ $\left[\left(x_{i}, y_{i}, z_{i}\right), l_{i}^{V}, \lambda_{i}^{V}, \beta_{i}^{V}\right]$ denotes a single segment and $f$ is the indicator function with $f\left(p_{i}, t_{1}, t_{2}, b, c\right)=1$ for segments that fulfil the conditions $1-3$ of the definition of $S_{D}$, otherwise $f=0$. In the following we describe the function $f$.

With $r_{1, i}^{A}+r_{2, i}^{A}=l_{i}^{V}$ it follows $0 \leq l_{i}^{V} \leq t_{1}+t_{2}$ as a first constraint. Furthermore we have $\beta_{i}^{V} \in[0, b]$ and $\lambda_{i}^{V} \in[0, c]$, therefore we choose

$$
\begin{aligned}
& f\left(p_{i}, t_{1}, t_{2}, b, c\right)= \\
& \quad \mathbb{1}_{\left[0, t_{1}+t_{2}\right]}\left(l_{i}^{V}\right) \mathbb{1}_{[0, b]}\left(\beta_{i}^{V}\right) \mathbb{1}_{[0, c]}\left(\lambda_{i}^{V}\right) \mathbb{1}_{Z_{0}}\left(x_{i}, y_{i}, z_{i}\right)
\end{aligned}
$$

for some set $Z_{0}=Z_{0}\left(\lambda_{i}^{V}, \beta_{i}^{V}, l_{i}^{V}, t_{1}, t_{2}\right)$ with $\left(x_{i}, y_{i}, z_{i}\right) \in$ $Z_{0}$ if and only if $p_{i}$ fulfils the conditions 1 and 3 . Let

$$
Z_{1}=D \oplus\left\{s \cdot e_{\lambda_{i}^{V}, \beta_{i}^{V}}, s \in\left[0, \min \left\{l_{i}^{V}, t_{1}\right\}\right]\right\}
$$

and

$$
Z_{2}=D \oplus\left\{s \cdot e_{\lambda_{i}^{V}, \beta_{i}^{V}}, s \in\left[l_{i}^{V}-\min \left\{l_{i}^{V}, t_{2}\right\}, l_{i}^{V}\right]\right\}
$$


with $e_{\lambda, \beta}=(\cos \lambda \sin \beta, \sin \lambda \sin \beta, \cos \beta)$, where $\oplus$ denotes the Minkowski addition. Then $p_{i}$ intersects the set $D$ with an upper segment length $r_{1, i}^{A} \leq t_{1}$ if and only if $\left(x_{i}, y_{i}, z_{i}\right) \in Z_{1}$ and $p_{i}$ intersects $D$ with a lower segment length $r_{2, i}^{A} \leq t_{2}$ if and only if $\left(x_{i}, y_{i}, z_{i}\right) \in Z_{2}$. In both cases $p_{i}$ intersects $D$ with the intersection angles $\beta_{i}^{V} \in[0, b]$ and $\lambda_{i}^{V} \in[0, c]$. It follows that $p_{i}$ fulfils the conditions 1 to 3 if and only if $\left(x_{i}, y_{i}, z_{i}\right) \in Z_{0}=Z_{1} \cap Z_{2}$. Due to the structure of $Z_{1}$ and $Z_{2}$ we can write $Z_{0}$ as the Minkowski sum of $D$ and a line-segment:

$$
\begin{aligned}
& Z_{0}= \\
& \quad D \oplus\left\{s \cdot e_{\lambda_{i}^{V}, \beta_{i}^{V}}, s \in\left[l_{i}^{V}-\min \left\{t_{2}, l_{i}^{V}\right\}, \min \left\{t_{1}, l_{i}^{V}\right\}\right]\right\} .
\end{aligned}
$$

Fig. 3 explains the underlying geometry.

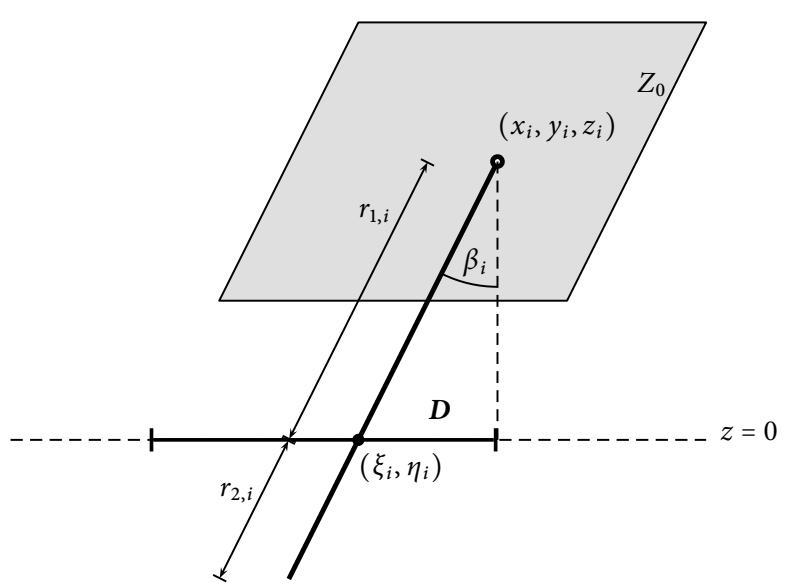

Figure 3. Underlying geometry in the $(x, z)$-plane of the intersection of a segment of azimuthal angle $\lambda_{i}^{V}=0$ with the plane $S$.

The right hand side of Eq. 13 can be calculated by means of the Campbell theorem. We obtain

$$
\begin{aligned}
& S_{D}\left(t_{1}, t_{2}, b, c\right)= \\
& =N_{V} \int_{\mathbb{R}^{3}\left[0, t_{1}+t_{2}\right] \times[0, b] \times[0, c]} \mathbb{1}_{Z_{0}}(x, y, z) . \\
& \mathrm{d} F_{V, L, B, \Lambda}(l, \beta, \lambda) \mathrm{d}(x, y, z) \\
& =N_{V} \int_{\mathbb{R}^{3}}^{t_{1}+t_{2}} \int_{0}^{b} \int_{0}^{b} \int_{0}^{c} \mathbb{1}_{Z_{0}}(x, y, z) \\
& f_{V, L, B, \Lambda}(l, \beta, \lambda) \mathrm{d} \lambda \mathrm{d} \beta \mathrm{d} l \mathrm{~d}(x, y, z) .
\end{aligned}
$$

By applying Fubini's theorem we can rearrange the order of integration, and we evaluate $\int \mathbb{1}_{Z_{0}}(x, y, z) \mathrm{d}(x, y, z)$ using Cavalieri's principle. Due $\mathbb{R}^{3}$ to the representation of $Z_{0}$ as a Minkowski sum we can express this integral by the product of the area of $D$, $v_{2}(D)$, and the height of $Z_{0}$. We get

$$
\begin{aligned}
& \int_{\mathbb{R}^{3}} \mathbb{1}_{Z_{0}}(x, y, z) d(x, y, z)= \\
& v_{2}(D) \cos \beta\left(\min \left\{l, t_{1}\right\}+\min \left\{l, t_{2}\right\}-l\right) .
\end{aligned}
$$

At this point the integrand is independent of $\lambda$. With Eq. 12 it follows the equivalence of the marginal distributions

$$
F_{A, \Lambda}(\lambda) \equiv F_{V, \Lambda}(\lambda) .
$$

Hence follows, we concentrate on the relation of the marginal distribution functions $F_{A, R_{1}, R_{2}, B}$ and $F_{V, L, B}$ and $S_{D}\left(t_{1}, t_{2}, b, 2 \pi\right)$. We obtain

$$
\begin{aligned}
& \quad S_{D}\left(t_{1}, t_{2}, b, 2 \pi\right)= \\
& \quad N_{V} \int_{\left[0, t_{1}+t_{2}\right] \times[0, b]} \cos \beta . \\
& \quad\left(\min \left\{l, t_{1}\right\}+\min \left\{l, t_{2}\right\}-l\right) \mathrm{d} F_{V, L, B}(l, \beta) .
\end{aligned}
$$

Equating Eq. 16 with Eq. 12 and applying integration by parts gives the desired relation Eq. 4 .

\section{APPLICATIONS AND DISCUSSION}

The stereological formulas Eqs. 6-9 can now be used to verify earlier results and to examine application-related cases.

\section{SUPERPOSITION OF LINE-SEGMENT PROCESSES}

In the following example the line-segments follow a special relation between length and direction: long fibres $\left(l \in\left[l_{0}, l_{\max }\right], 0<l_{0}<l_{\max }\right)$ all have the same polar angle $\beta_{0} \in\left[0, \frac{\pi}{2}\right]$ while short fibres $\left(l \in\left[0, l_{0}\right]\right)$ are isotropic. The proportion of segments of length $l \in\left[0, l_{0}\right]$ is $p \in[0,1]$ and for segments with $l \in\left[l_{0}, l_{\max }\right]$ is $1-p$. The joint probability density function of $L^{V}$ and $B^{V}$ is therefore

$$
f_{V, L, B}(l, \beta)= \begin{cases}\frac{p}{l_{0}} \sin \beta, & l \leq l_{0} \\ \frac{1-p}{l_{\max }-l_{0}} \delta_{\beta_{0}}(\beta), & l_{0} \leq l \leq l_{\max } \\ 0, & l>l_{\max } .\end{cases}
$$

Thus, this example represents a superposition of two different line-segment processes. With Eq. 6 the mean number of section points per unit area is

$$
N_{A}=N_{V}\left(\frac{1}{4} p l_{0}+\frac{1}{2} \cos \beta_{0}(1-p)\left(l_{\max }+l_{0}\right)\right) \text {. }
$$


Moreover using Eq. 7 and Eq. 8 the conditional distribution function

$$
\begin{aligned}
& F_{A, R_{1} \mid R_{2} \leq r_{2}}\left(r_{1}\right)= \\
& \left(\int _ { 0 } ^ { \operatorname { m i n } \{ r _ { 1 } , r _ { 2 } \} } \int _ { 0 } ^ { \frac { \pi } { 2 } } \operatorname { s i n } \beta ^ { \prime } \left(F_{V, L, B}\left(l+\max \left\{r_{1}, r_{2}\right\}, \beta^{\prime}\right)\right.\right. \\
& \left.\left.\quad-F_{V, L, B}\left(l, \beta^{\prime}\right)\right) \mathrm{d} \beta^{\prime} \mathrm{d} l\right) / \\
& \left(\int_{0}^{r_{2}} \int_{0}^{\frac{\pi}{2}} \sin \beta^{\prime}\left(F_{V, B}\left(\beta^{\prime}\right)-F_{V, L, B}\left(l, \beta^{\prime}\right)\right) \mathrm{d} \beta^{\prime} \mathrm{d} l\right)
\end{aligned}
$$

as well as the conditional density function

$$
\begin{aligned}
& f_{A, R_{1} \mid R_{2} \leq r_{2}}\left(r_{1}\right)= \\
& \frac{\int_{0}^{\frac{\pi}{2}} \sin \beta^{\prime}\left(F_{V, L, B}\left(r_{1}+r_{2}, \beta^{\prime}\right)-F_{V, L, B}\left(r_{1}, \beta\right)\right) \mathrm{d} \beta^{\prime}}{\int_{0}^{r_{2}} \int_{0}^{\frac{\pi}{2}} \sin \beta^{\prime}\left(F_{V, B}\left(\beta^{\prime}\right)-F_{V, L, B}\left(l, \beta^{\prime}\right)\right) \mathrm{d} \beta^{\prime} \mathrm{d} l}
\end{aligned}
$$

can be calculated. The conditional probability density function $f_{A, R_{1} \mid R_{2} \leq r_{2}}\left(r_{1}\right)$ is shown in Fig. 4 for $l_{0}=1$, $l_{\max }=3, \beta_{0}=\frac{3}{4}$ and $p=\frac{3}{4}$ and by means of Eq. 18 the ratio $\frac{N_{A}}{N_{V}}$ is 0.553 . Thus, $N_{A}$ can be determined once $N_{V}$ is known.

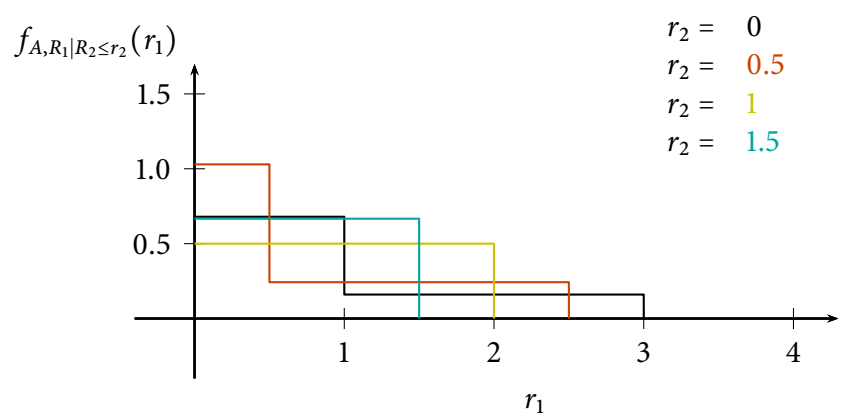

Figure 4. The conditional probability density function $f_{A, R_{1} \mid R_{2} \leq r_{2}}\left(r_{1}\right)$ of the upper segment length $R_{1}^{A}$ under the condition the lower segment length $R_{2}^{A}$ is bounded from above.

\section{INDEPENDENT FIBRE ANGLES AND LENGTHS}

Assume the line-segment length $L^{V}$ is

\begin{tabular}{|c|c|}
\hline$N_{V}$ & mean number of top points of \\
\hline$F_{V, L}(l)$ & $\begin{array}{l}\text { line segments per unit volume } \\
\text { distribution function of length } \\
\text { of a typical segment of } \Psi_{V}\end{array}$ \\
\hline$F_{V, \Lambda}(\lambda), F_{V, B}(\beta)$ & $\begin{array}{l}\text { distribution function of azimuth } \\
\text { and polar angle of a typical } \\
\text { segment of } \Psi_{V}\end{array}$ \\
\hline
\end{tabular}
stochastically independent of the angles $\Lambda^{V}$ and $B^{V}$ for the line-segment process $\Psi_{V}$. Under this assumption this line-segment process can be characterized using the following parameters and functions:
Table 3. Characteristics of the spatial marked point process $\Psi_{V}$ under the assumption that $L^{V}$ and $B^{V}$ are stochastically independent.

Eq. 4 simplifies in the following way:

$$
\begin{aligned}
& N_{A} F_{A, R_{1}, R_{2}, B}\left(r_{1}, r_{2}, \beta\right)= \\
&=N_{V} \int_{0}^{\min \left\{r_{1}, r_{2}\right\}} \int_{0}^{\beta}\left(F_{V, L}\left(l+\max \left\{r_{1}, r_{2}\right\}\right)-F_{V, L}(l)\right) \\
& \cdot \cos \beta^{\prime} \mathrm{d} F_{V, B}\left(\beta^{\prime}\right) \mathrm{d} l \\
&=N_{V} \int_{0}^{\min \left\{r_{1}, r_{2}\right\}}\left(F_{V, L}\left(l+\max \left\{r_{1}, r_{2}\right\}\right)-F_{V, L}(l)\right) \mathrm{d} l \\
& \int_{0} \sin \beta^{\prime} F_{V, B}\left(\beta^{\prime}\right) \mathrm{d} \beta^{\prime}
\end{aligned}
$$

for $r_{1}, r_{2}>0, \beta \in\left[0, \frac{\pi}{2}\right]$ and $N_{A}, N_{V}>0$. It follows that the segment lengths $R_{1}^{A}, R_{2}^{A}$ and the polar angle $B^{A}$ of the segment process $\Psi_{A}$ are stochastically independent.

We therefore concentrate on the marginal distribution functions $F_{A, R_{1}, R_{2}}\left(r_{1}, r_{2}\right)$ and $F_{A, B}(\beta)$. We have

$$
\begin{gathered}
N_{A} F_{A, R_{1}, R_{2}}\left(r_{1}, r_{2}\right) F_{A, B}(\beta)= \\
N_{V} \int_{0}^{\min \left\{r_{1}, r_{2}\right\}}\left(F_{V, L}\left(l+\max \left\{r_{1}, r_{2}\right\}\right)-F_{V, L}(l)\right) \mathrm{d} l . \\
\int_{0}^{\beta} \sin \beta^{\prime} F_{V, B}\left(\beta^{\prime}\right) \mathrm{d} \beta^{\prime}
\end{gathered}
$$

Using this simplified relation we obtain

$$
\begin{aligned}
& N_{A}=N_{V} \mathbf{E} L^{V} \mathbf{E} \cos B^{V} \\
& F_{A, R_{1}, R_{2}}\left(r_{1}, r_{2}\right)= \\
& \frac{1}{\mathbf{E} L^{V}} \int_{0}^{\min \left\{r_{1}, r_{2}\right\}}\left(F_{V, L}\left(l+\max \left\{r_{1}, r_{2}\right\}\right)-F_{V, L}(l)\right) \mathrm{d} l
\end{aligned}
$$


and

$$
F_{A, B}(\beta)=\frac{1}{\mathbf{E} \cos B^{V}} \int_{0}^{\beta} \sin \beta^{\prime} F_{V, B}\left(\beta^{\prime}\right) \mathrm{d} \beta^{\prime} .
$$

The segment lengths $R_{1}^{A}$ and $R_{2}^{A}$ are identically distributed, i.e., the marginal distributions $F_{A, R_{1}}$ and $F_{A, R_{2}}$ are equivalent:

$$
\begin{aligned}
F_{A, R}(r)=F_{A, R_{1}}(r)=F_{A, R_{2}}(r)= & \\
& \frac{1}{\mathbf{E} L^{V}} \int_{0}^{r}\left(1-F_{V, L}(l)\right) \mathrm{d} l, \quad r>0 .
\end{aligned}
$$

Concerning the corresponding probability density functions of $\Psi_{V}$ and $\Psi_{A}$ it holds

$$
f_{A, R_{1}, R_{2}}\left(r_{1}, r_{2}\right)=\frac{1}{\mathbf{E} L^{V}} f_{V, L}\left(r_{1}+r_{2}\right),
$$

and

$$
f_{A, B}(\beta)=\frac{1}{\mathbf{E} \cos B^{V}} f_{V, B}(\beta) \cos \beta .
$$

\section{THE ISOTROPIC CASE}

We assume that the line-segment length $L^{V}$ is stochastically independent of the angles $\Lambda^{V}$ and $B^{V}$ for the line-segment process $\Psi_{V}$. Furthermore the linesegment process $\Psi_{V}$ is assumed to be isotropic, i.e., the directional vector of the typical line-segment is uniformly distributed on the unit sphere. We have

$$
f_{V, B}(\beta)=\sin \beta,
$$

and the distribution function

$$
F_{V, B}(\beta)=1-\cos \beta
$$

for this case. Eq. 9 yields

$$
f_{A, B}(\beta)=\sin 2 \beta,
$$

and

$$
F_{A, B}(\beta)=\sin ^{2} \beta,
$$

which is a result true for general fibre processes, see Mecke and Nagel (1980).

With Eq. 6 we obtain $N_{A}$, the mean number of section points per unit area as

$$
N_{A}=\frac{1}{2} N_{V} \mathbf{E} L^{V} .
$$

This result is a special case of another well-known stereological formula, namely (11.3.3) in Chiu et al. (2013), where characteristics of germ-grain models are studied. Here the "grain" is an isotropic line-segment.

\section{SEGMENTS OF CONSTANT LENGTH}

In papers such as Li et al. (1991) and Brandt (1985) line-segment processes and their sections with planes are studied. They derived formulas for the strength of fibre-reinforced materials using calculations for segments of constant length. If in this context the constant length is chosen to be $l_{0}>0$, we obtain with Eq. 8 the marginal distribution function $F_{A, R}(r)$. It holds $F_{V, L}(l)=\Theta\left(l-l_{0}\right)$ with the Heaviside function

$$
\Theta(x)= \begin{cases}0, & x<0 \\ 1, & x \geq 0\end{cases}
$$

and

$$
\begin{aligned}
F_{A, R}(r) & =\frac{1}{l_{0}} \int_{0}^{r}\left(1-\Theta\left(l-l_{0}\right)\right) \mathrm{d} l \\
& = \begin{cases}\frac{r}{l_{0}}, & r \leq l_{0} \\
1, & r \geq l_{0}\end{cases}
\end{aligned}
$$

for the distribution function of the upper and lower segment length at a typical section point. This distribution is the uniform distribution on $\left[0, l_{0}\right]$. We therefore obtain the mean values of the residual segment lengths $\mathbf{E} R_{1}^{A}=\mathbf{E} R_{2}^{A}=\frac{1}{2} l_{0}$. This result coincides with results of Li et al. (1991).

Moreover the line-segments in (Li et al., 1991) are considered to be isotropic. Using Eq. 26 we have $N_{A}=\frac{1}{2} N_{V} l_{0}$.

\section{A PARAMETRIC MODEL}

In Chin et al. (1988), Hegler (1985) and other papers line-segment length and angle distribution functions for segment processes $\Psi_{V}$ with $L^{V}$ independent of $B^{V}$ appear such as

$$
F_{V, L}(l)=1-\mathrm{e}^{-(m l)^{k}}, \quad m, k, l>0,
$$

the Weibull distribution function, and

$$
F_{V, B}(\beta)=\frac{1-\exp (-\eta \beta)}{1-\exp \left(-\frac{\pi}{2} \eta\right)}, \quad \beta \in\left[0, \frac{\pi}{2}\right], \eta>0 .
$$

These distribution functions are motivated by the process of manufacture of some fibre-reinforced material. They model different effects such as breakage of fibres before moulding or a preferred orientation of fibres after moulding, where the shape parameter $\eta$ models the orientation density of the linesegments. A large $\eta$ indicates a major preferential alignment of the line-segments in the $z$-direction, see also Kacir et al. (1975) and Chin et al. (1988). Note that this parametric model does not include the 
isotropic case. The Figs. 5 and 6 give an impression of the influence of the parameters $m, k$ and $\eta$ on the probability density functions $f_{V, L}$ and $f_{V, B}$.

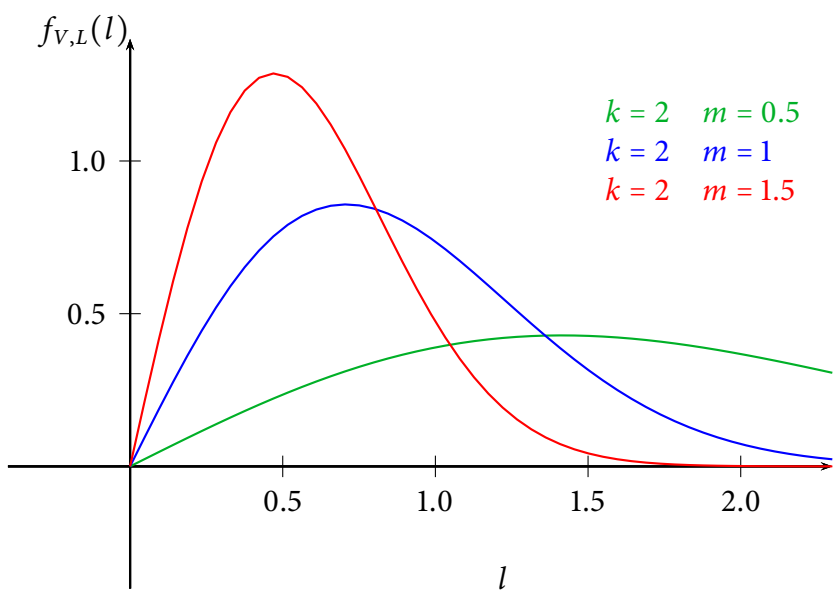

Figure 5. Influence of the parameters $k$ and $m$ on the Weibull probability densitiy function of $L^{V}$.

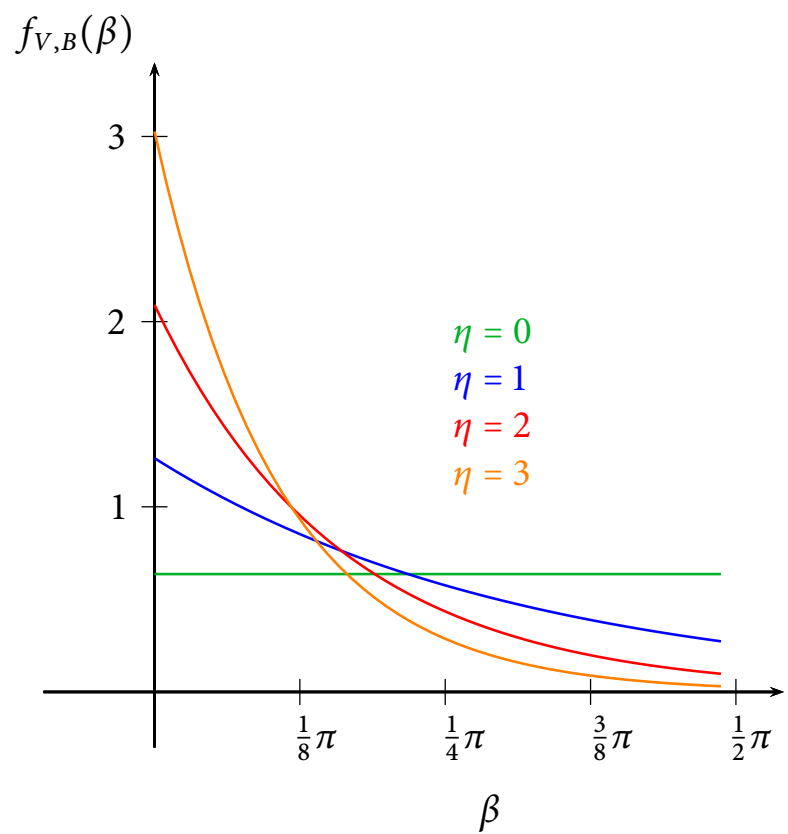

Figure 6. Influence of the shape parameter $\eta$ on the probability density function of polar angle $B^{V}$.

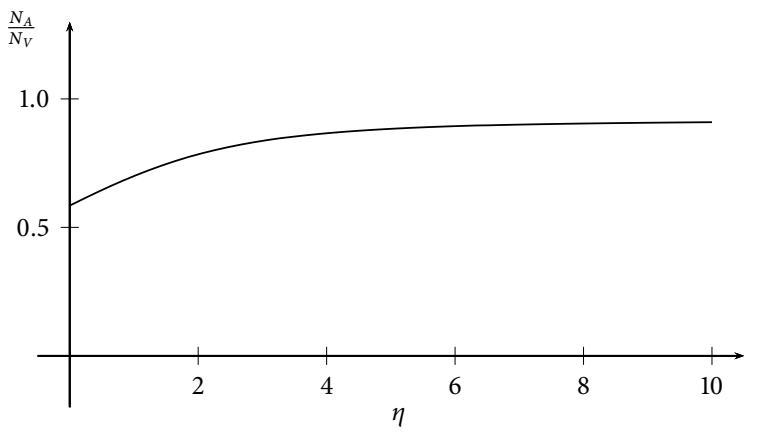

Figure 7. The ratio $\frac{N_{A}}{N_{V}}$ in dependence on shape parameter $\eta$.

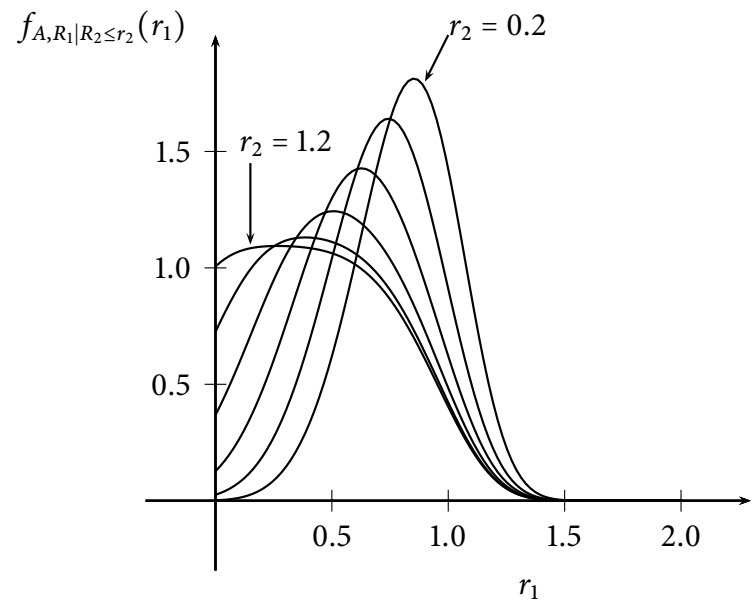

Figure 8 . The conditional probability density function $f_{A, R_{1} \mid R_{2} \leq r_{2}}\left(r_{1}\right)$ in dependence on $r_{2}$ for Weibulldistributed fibre lengths. This is the probability density function of the length $R_{1}^{A}$ of the segment above the plane $S$, if the segment length $R_{2}^{A}$ below $S$ is bounded from above. Here $r_{2}$ ranges from 0.2 to 1.2 with a step size of 0.2.

The distribution of the residual segment lengths can be easily computed using the Eqs. 6-9. For the parameters $m=1$ and $k=5$ the results are shown in Figs. 7-9.

Fig. 7 show that $\frac{N_{A}}{N_{V}}$ goes to a limit value as $\eta$ tends to infinity. This limit is $\mathbf{E} L^{V}$, the mean length of the line-segments (in the case $m=1$ and $k=5$ we have the limit value $\mathbf{E} L^{V} \approx 0.918$ ). This is plausible since for large $\eta$ the segments have a preferred orientation in $z$-direction. Therefore with $\eta \rightarrow \infty$ all line-segments have the polar angle $B^{V}=0$ and therefore $B^{A}=0$. Applying this case to Eq. 6 we find $N_{A}=N_{V} \mathbf{E} L^{V}$.

Furthermore, Fig. 8 shows that the conditional distribution of the upper segment length

$$
f_{A, R_{1} \mid R_{2} \leq r_{2}}\left(r_{1}\right)=\frac{F_{V, L}\left(r_{1}+r_{2}\right)-F_{V, L}\left(r_{1}\right)}{\int_{0}^{r_{2}}\left(1-F_{V, L}(l)\right) \mathrm{d} l}
$$

is more concentrated if the lower segment length is fixed at small values. Moreover it can be shown that $f_{A, R_{1} \mid R_{2} \leq r_{2}}\left(r_{1}\right)$ coincides with $f_{V, L}(l)$ if $r_{2} \rightarrow 0$. Thus with decreasing upper bound of $R_{2}^{A}$ the conditional probability density function in Fig. 8 tends to the probability density function of the Weibull distribution with $m=1$ and $k=5$ in Fig. 8. With increasing $r_{2}$ the conditional probability $f_{A, R_{1} \mid R_{2} \leq r_{2}}\left(r_{1}\right)$ tends to $\frac{1}{\mathbf{E} L^{V}}\left(1-F_{V, L}\left(r_{1}\right)\right)$. Since the segment lengths $R_{1}^{A}$ and $R_{2}^{A}$ are independent of the intersection angle $B^{A}$ in this case the conditional probability density function $f_{A, R_{1} \mid R_{2} \leq r_{2}}$ is independent of $\eta$. 


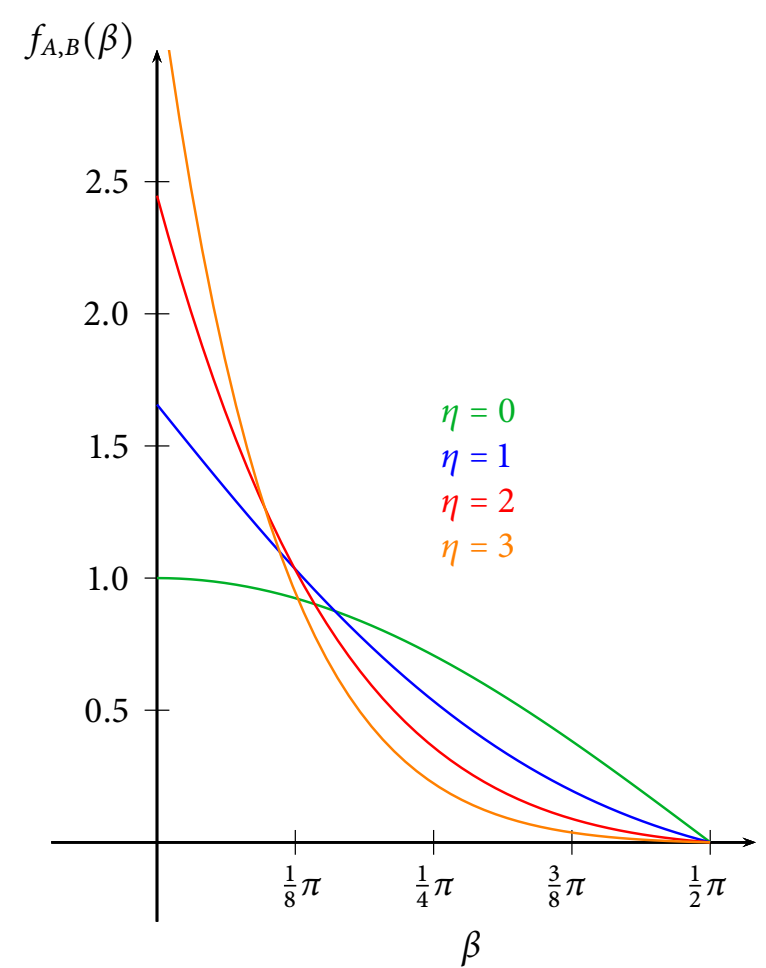

Figure 9. The probability density function $f_{A, B}(\beta)$ of intersection angles in dependence on orientation distribution parameter $\eta$ which varies from 0 to 3 .

In Fig. 9 we see that with increasing $\eta$ the segments at the typical section point show a preferred direction in the $z$-axis. Since the segment lines tend to have a small polar angle $B^{V}$ for large $\eta$, the segments intersecting $S$ have a small polar angle $B^{A}$ too. For $\eta \rightarrow 0$ the probability density function $f_{A, B}(\beta)$ tends to $\cos \beta$. Note that this is not the isotropic case since for isotropic line-segments it holds $f_{A, B}(\beta)=\sin 2 \beta$.

\section{MAXIMUM AND MINIMUM RESIDUAL SEGMENT LENGTH}

We investigate the stochastic variables $M=$ $\max \left\{R_{1}^{A}, R_{2}^{A}\right\}$ and $m=\min \left\{R_{1}^{A}, R_{2}^{A}\right\}$, which are only in special cases stochastic independent. We therefore consider first the joint distribution function $F_{A, m, M}\left(r_{m}, r_{M}\right)$ with $r_{m}, r_{M}>0$. Furthermore we consider the marginal distribution functions

$$
\begin{aligned}
& F_{A, M}(r)=P(M \leq r)=P\left(R_{1}^{A} \leq r, R_{2}^{A} \leq r\right) \quad \text { and } \\
& F_{A, m}(r)=P(m \leq r)=1-P\left(R_{1}^{A}>r, R_{2}^{A}>r\right)
\end{aligned}
$$

for $r>0$. Using simple ideas of probability we obtain

$$
\begin{aligned}
& F_{A, m, M}\left(r_{m}, r_{M}\right)=\left\{\begin{array}{cc}
F_{A, R_{1}, R_{2}}\left(r_{m}, r_{M}\right) & \\
+F_{A, R_{1}, R_{2}}\left(r_{M}, r_{m}\right) & r_{m} \leq r_{M} \\
-F_{A, R_{1}, R_{2}}\left(r_{m}, r_{m}\right) & \\
& \\
F_{A, R_{1}, R_{2}}\left(r_{M}, r_{M}\right) & r_{M} \leq r_{m}
\end{array}\right. \\
& F_{A, M}(r)=F_{A, R_{1}, R_{2}}(r, r)
\end{aligned}
$$

and

$$
F_{A, m}(r)=2 F_{A, R}(r)-F_{A, R_{1}, R_{2}}(r, r) .
$$

With Eqs. 7 and 8 we can relate these distribution functions with the distribution functions of $\Psi_{V}$ :

$$
\begin{aligned}
& F_{A, m, M}\left(r_{m}, r_{M}\right)= \\
& \frac{1}{\mathbf{E} L^{V}} \int_{0}^{r_{m}}\left(2 F_{V, L}\left(l+r_{M}\right)-F_{V, L}\left(l+r_{m}\right)-F_{V, L}(l)\right) \mathrm{d} l \\
& F_{A, M}(r)= \\
& \frac{1}{\mathbf{E} L^{V}} \int_{0}^{r}\left(F_{V, L}(l+r)-F_{V, L}(l)\right) \mathrm{d} l \\
& F_{A, m}(r)= \\
& \frac{1}{\mathbf{E} L^{V}} \int_{0}^{r}\left(2-F_{V, L}(l)-F_{V, L}(l+r)\right) \mathrm{d} l
\end{aligned}
$$

The marginal distributions simplify with $F_{V, \bar{L}}(l)=1-$ $F_{V, L}(l)$ :

$$
F_{A, M}(r)=\frac{1}{\mathbf{E} L^{V}} \int_{0}^{r}\left(F_{V, \bar{L}}(l)-F_{V, \bar{L}}(l+r)\right) \mathrm{d} l
$$

and

$$
F_{A, m}(r)=\frac{1}{\mathbf{E} L^{V}} \int_{0}^{r}\left(F_{V, \bar{L}}(l)+F_{V, \bar{L}}(l+r)\right) \mathrm{d} l .
$$

In case of Weibull-distributed lengths it holds $F_{V, L}(l)=$ $1-\mathrm{e}^{-(m l)^{k}}$ and $F_{V, \bar{L}}(l)=\mathrm{e}^{-(m l)^{k}}$. The corresponding results are sketched in Fig. 10.

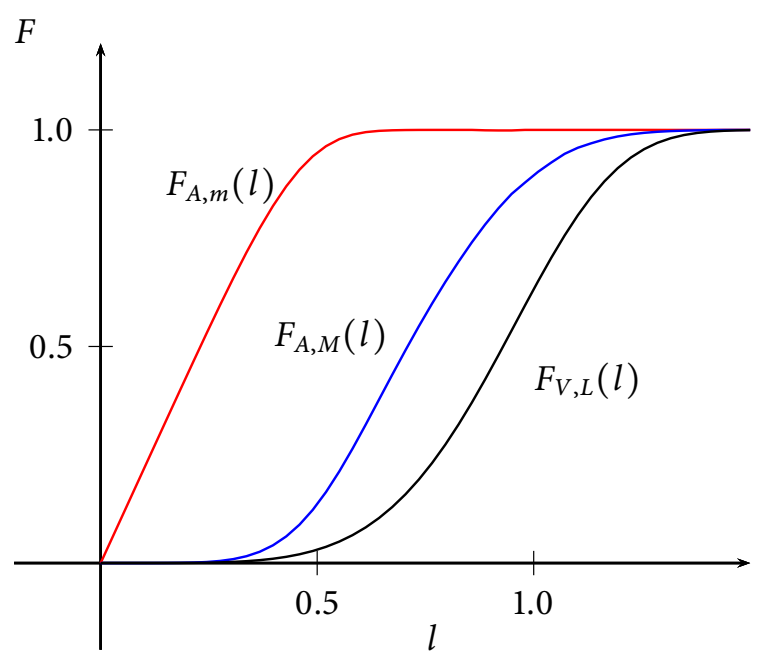

Figure 10. Distribution functions of minimum and maximum residual length, $F_{A, m}(l)$ and $F_{A, M}(l)$, for Weibull-distributed total lengths with $m=1$ and $k=5$. 


\section{ACKNOWLEDGEMENTS}

The authors thank K.G. van den Boogaart for inspiring discussion during a seminar at the Institute of Stochastics of the TU Freiberg and U. Hampel, M. Bieberle and S. Boden for providing CT data of fibre-reinforced autoclaved aerated concrete. They are also grateful to the reviewers for series of valuable comments.

\section{REFERENCES}

Brandt AM (1985). On the optimal direction of short metal fibres in brittle matrix composites. J Mater Sci 20:383141.

Carling MJ, Williams JG (1990). Fiber length distribution effects on the fracture of short-fiber composites. Polymer Composites 11:307-13.

Chin WK (1988). Effects of fiber length and orientation distribution on the elastic modulus of short fiber reinforced thermoplastics. Polymer Composites 9:2735.

Chiu SN, Stoyan D, Kendall WS, Mecke J (2013). Stochastic geometry and its applications. 3rd Ed. Chichester: Wiley.

Fu SY, Lauke B (1996). Effects of fiber length and fiber orientation distributions on the tensile strength of shortfiber-reinforced polymers. Comp Sci Tech 56:1179-90.

Hegler RP (1985). Phase separation effects in processing of glass-bead- and glass-fiber-filled thermoplastics by injection molding. Poly Eng Sci 25:395-405.

Kacir L, Narkis N, Ishai O (1975). Oriented short glass- fiber composites. I. preparation and statistical analysis of aligned fiber mats. Poly Eng Sci 15:525-31.

Li VC, Wang Y, Backer S (1991). A micromechanical model of tension softening and bridging toughening of short random fiber reinforced brittle matrix composites. J Mech Phys Solids 39:607-25.

Maalej M, Li VC, Hashida T (1995). Effect of fibre rupture on tensile properties of short fibre composites. J Eng Mech 121:903-13.

Mecke J, Nagel W (1980). Stationäre räumliche Faserprozesse und ihre Schnittzahlrosen. Elektron Informationsverarb Kyb 16:475-83.

Mecke J, Stoyan D (1980a). A general approach to Buffon's needle and Wicksell's corpuscle problem. In: Combinatorial principles in stochastic geometry, Work Collect. Erevan. 164-71.

Mecke J, Stoyan D (1980b). Formulas for stationary planar fibre processes I - general theory. Math Operationsforsch Statist Ser Statist 11:267-79.

Mecke J, Stoyan D (1980c). Stereological problems for spherical particles. Math Nach 96:311-7.

Naaman AE, Reinhardt HW (1996). High performance fiber reinforced cement composites 2 (HPFRCC2). In: Proc 2nd Int RILEM Worksh.

Vaxman A, Narkis M, Siegmann A (1989). Short-fiberreinforced thermoplastics. Part III: Effect of fiber length on rheological properties and fiber orientation. Polymer Composites 10:454-62.

Wanga C, Friedrich LF (2013). Computational model of spalling and effective fibre on toughening in fiber reinforced composites at an early stage of crack formation. Lat Am J Solids Struct 10:707-811. 